\title{
Vertical diffusivities of active and passive tracers
}

\author{
V.M. Canuto ${ }^{\mathrm{a}, \mathrm{b}, *}$, Y. Cheng ${ }^{\mathrm{a}, \mathrm{c}}$, A.M. Howard ${ }^{\mathrm{a}, \mathrm{d}}$ \\ ${ }^{a}$ NASA, Goddard Institute for Space Studies, New York, NY 10025, USA \\ ${ }^{\mathrm{b}}$ Department of Applied Physics and Applied Mathematics, Columbia University, New York, NY 10027, USA \\ ${ }^{\mathrm{c}}$ Center for Climate Systems Research, Columbia University, New York, NY 10025, USA \\ ${ }^{\mathrm{d}}$ Department of Physical, Environmental and Computer Science, Medgar Evers College, CUNY, NY 11225, USA
}

\section{A R T I C L E I N F O}

\section{Article history:}

Received 28 June 2010

Received in revised form 1 December 2010

Accepted 4 December 2010

Available online 15 December 2010

\section{Keywords:}

Diffusivities

Tracers

OGCM

Turbulence

\begin{abstract}
A B S T R A C T
The climate models that include a carbon-cycle need the vertical diffusivity of a passive tracer. Since an expression for the latter is not available, it has been common practice to identify it with that of salt. The identification is questionable since $T, S$ are active, not passive tracers. We present the first derivation of the diffusivity of a passive tracer in terms of Ri (Richardson number) and $R_{\rho}$ (density ratio, ratio of salinity over temperature $z$-gradients). The following results have emerged:
\end{abstract}

(a) The passive tracer diffusivity is an algebraic function of $R i, R_{\rho}$.

(b) In doubly stable regimes (DS, $\partial T / \partial z>0, \partial S / \partial z<0$ ), the passive scalar diffusivity is nearly the same as that of salt/heat for any values of $R_{\rho}<0$ and $R i>0$.

(c) In DC regimes (diffusive convection, $\partial T / \partial z<0, \partial S / \partial z<0, R_{\rho}>1$ ), the passive scalar diffusivity is larger than that of salt. At $R i=O(1)$, it can be more than twice as large.

(d) In $S F$ regimes (salt fingers, $\partial T / \partial z>0, \partial S / \partial z>0, R_{\rho}<1$ ), the passive scalar diffusivity is smaller than that of salt. At $R i=\mathrm{O}(1)$, it can be less than half of it.

(e) The passive tracer diffusivity predicted at the location of NATRE (North Atlantic Tracer Release Experiment) is discussed.

(f) Perhaps the most relevant conclusion is that the common identification of the tracer diffusivity with that of salt is valid only in DS regimes. In the Southern Ocean, where there is the largest $\mathrm{CO}_{2}$ absorption, the dominant regime is diffusive convection discussed in (c) above.

Published by Elsevier Ltd.

\section{Formulation of the problem}

Since ocean general circulation models (OGCMs) cannot resolve small scale turbulent processes, the latter must be parameterized in terms of the resolved fields. OGCMs solve the time dependent equations for the mean velocity, mean temperature, mean salinity and mean concentration of passive tracers such as $\mathrm{CO}_{2}$, since the carbon cycle is an important diagnostic in climate studies. Some climate studies also include CFC. While both temperature and salinity are active tracers, for they affect the velocity field via the buoyancy term and thus alter the status of the turbulent flow, tracers such as $\mathrm{CO}_{2}, \mathrm{CFC}$, Oxygen, if sufficiently diluted, as is ordinarily the case in the ocean, are passively carried along by the flow without altering it. ${ }^{1}$ It follows that the diffusivity of a passive tracer must

* Corresponding author at: NASA, Goddard Institute for Space Studies, New York, NY 10025, USA. Tel.: +1 212 6785571; fax: +1 2126785560 .

E-mail address: vcanuto@giss.nasa.gov (V.M. Canuto).

1 As pointed out by a referee, there are cases of lakes in which the concentrations of $\mathrm{CO}_{2}$ and $\mathrm{CH}_{4}$ contribute significantly to the density stratification to give rise to double-diffusive convection (Schmid et al., 2010). be different from that of heat and salt. Identifying the passive tracer diffusivity with that of salt is, however, a common practice probably because no expression for a passive tracer diffusivity is available in the literature.

In a recent work (Canuto et al., 2010, C10), ${ }^{2}$ we derived the vertical diffusivities for momentum, heat and salt as functions of the two large scale parameters $R i$ and $R_{\rho}$ (Richardson number and the density ratio) defined as follows:

$R i=\frac{N^{2}}{\Sigma^{2}}, \quad R_{\rho}=\frac{\beta \bar{S}_{, z}}{\alpha \bar{T}_{, z}}$

where $\Sigma=\left(2 S_{i j} S_{i j}\right)^{1 / 2}$ is the mean shear with $2 S_{i j}=\bar{U}_{i, j}+\bar{U}_{j, i}$ with $i$, $j=1,2,3 ; \bar{T}, \bar{S}$ and $\overline{\mathbf{U}}$ are the mean potential temperature, mean salinity and mean velocity fields; we employ the notation $a_{, z} \equiv \partial a /$ $\partial z$. The thermal expansion and haline contraction coefficients $\alpha, \beta=\left(-\rho^{-1} \partial \rho / \partial \bar{T},+\rho^{-1} \partial \rho / \partial \bar{S}\right)$ are computed using the non-linear UNESCO equation of state (UNESCO, 1981). The density ratio $R_{\rho}$

\footnotetext{
${ }^{2}$ In order to make this presentation self-contained, in Appendix A we report the expressions derived in $\mathrm{C} 10$ which are needed here.
} 
accounts for double-diffusion (DD) processes such as SF-salt fingers, $0<R_{\rho}<1$ (warm-salty water over cold-fresh water), DC-diffusive convection, $R_{\rho}>1$ (cold-fresh water over warm-salty water) and DS-doubly stable $R_{\rho}<0$ (warm-fresh water over cold-salty water). Specifically, in C10 it was shown that the solutions of the Reynolds stress model (RSM) yield the following expressions for the diffusivities $K_{\alpha}$ where the subscript $\alpha$ stands for momentum, heat and salt:

$K_{\alpha}=\frac{2 K^{2}}{\varepsilon} S_{\alpha}=\Gamma_{\alpha} \frac{\varepsilon}{N^{2}}, \quad \Gamma_{\alpha}=\frac{1}{2} S_{\alpha}(\tau N)^{2}$

Here, $K$ is the eddy kinetic energy, $\varepsilon$ is its rate of dissipation, $\tau=2 \mathrm{K \varepsilon}^{-1}$ is the dynamical time scale and $N$ is the Brunt-Vaisala frequency, $N^{2}=-g \rho_{0}^{-1} \partial \bar{\rho} / \partial z$. The functions $\Gamma_{\alpha}$ are known as mixing efficiencies and $S_{\alpha}$ are dimensionless structure functions both of which are given by the RSM as functions of:

$\Gamma_{\alpha}\left[R i, R_{\rho},(\tau N)^{2}\right], \quad S_{\alpha}\left[R i, R_{\rho},(\tau N)^{2}\right]$

In the mixed layer the first representation in (2) is more convenient since one can use a $K-\varepsilon$ model to determine these two variables while in the thermocline the $\varepsilon$-equation is quite uncertain and the second representation in (2) is more convenient. In fact, IGW (internal gravity waves) studies (Polzin et al., 1995; Polzin, 1996; Kunze and Sanford, 1996; Gregg et al., 1996; Toole, 1998), provide an expression for $\varepsilon N^{-2}$. The task of the turbulence models is that of modeling the mixing efficiencies $\Gamma_{\alpha}\left(R i, R_{\rho}\right)$.

Since in $\mathrm{C} 10$ we did not derive an expression for the diffusivity of a passive tracer, here we remedy that shortcoming by presenting a derivation for such a diffusivity.

The derivation is not trivial not because of mathematical but rather physical reasons and that is why we include it in the main text rather than in an Appendix. The difficulties are of two kinds. First, since the starting Eq. (4) is valid for an arbitrary tracer, during the derivation one must identify where and how a generic tracer is treated as passive or active. This is a somewhat delicate problem but not a very difficult one. The most difficult part is the determination of the dissipation time scales that characterize small scale processes in the passive tracer case. In $\mathrm{C} 10$, we presented a lengthy discussion of the analogous difficulty when one treats active tracers such as temperature and salinity. Assuming a constant ratio of the dissipation time scales to the dynamical time scale, as it was assumed for many years, ultimately because lack of data did not motivate other suggestions, yielded a rather poor fit to the NATRE data (North Atlantic Tracer Release Experiment, Ledwell et al., 1993, 1998; St. Laurent and Schmitt, 1999, see Fig. 3 of Canuto et al., 2008). A better fit was achieved when the time scale ratios were made $R i, R_{\rho}$ dependent. Within the present context, the question is how to relate those dissipation times scales to the new ones that characterize a passive tracer. As we discuss below, the second part of the derivation consists of finding such a relation which we believe we have done without introducing arbitrary or heuristic assumptions. Rather, we required that the diffusivity that we obtain with a direct solution of the turbulent equation for a passive scalar, ought to coincide with those of either salinity and/or temperature when the latter are considered so diluted as not to affect the density field and thus behave like passive tracers. Such a requirement allows to find a relation between the dissipation time scale of a passive tracer and those already determined in the temperature-salinity case.

\section{Passive tracer vertical flux $J_{c}=\overline{w^{\prime} c^{\prime}}$}

We begin with the general equation for an arbitrary tracer (often referred to as a scalar) which at this point can be either passive or active. Such an equation is $\partial_{t} \rho c+\partial_{i}\left(\rho c u_{i}\right)=\chi_{c} \rho \partial_{i}^{2} c$

where the rhs represents molecular effects with a kinematic diffusivity $\chi_{c}$. Following standard procedure, the total field $\mathrm{c}$ is split into an average and a fluctuating component, $c=\bar{C}+c^{\prime}$ and the same is done with the total velocity field $u_{i}=\bar{U}_{i}+u_{i}^{\prime}$. Next, we introduce the above decompositions in (4), use the incompressibility condition, average the result and subtract it from the equation for the total c-field. These purely algebraic steps lead to the following equation for the fluctuating component of the generic field $c^{\prime}\left(D_{t}=\partial_{t}+\bar{U}_{i} \partial_{i}\right)$ :

$D_{t} c^{\prime}+u_{i}^{\prime} \partial_{i} \bar{c}+\partial_{i}\left(u_{i}^{\prime} c^{\prime}-\overline{u_{i}^{\prime} c^{\prime}}\right)=\chi_{c} \partial_{i}^{2} c^{\prime}$

Using an analogous procedure for the velocity field described by the Navier-Stokes equations, one derives the equation for the field $w^{\prime}$ which reads as follows:

$\partial_{t} w^{\prime}=-\rho_{0}^{-1} \partial_{z} p^{\prime}-g \rho_{0}^{-1} \rho^{\prime}-\partial_{j}\left(w^{\prime} u_{j}^{\prime}-\overline{w^{\prime} u_{j}^{\prime}}\right)+v \partial_{i}^{2} w^{\prime}$

where $v$ is the kinematic viscosity of seawater. As expected, the averages of both (5)-(6) yield the identity $0=0$ since all fluctuating variables have zero average.

Next, we recall that if the field $c$ is not affecting the density, and this is the assumption about $c$ being a passive tracer, then the density is only affected by the truly active scalars, temperature and salinity and thus the density field $\rho^{\prime}$ in (6a) is taken to be:

$\rho^{\prime} \rho_{0}^{-1}=-\alpha \theta^{\prime}+\beta s^{\prime}$

where $\theta^{\prime}, s^{\prime}$ are the fluctuating components of the $T$ and $S$ fields. The next step is again purely algebraic and consists of multiplying Eq. (5) by $w^{\prime}$ and Eq. (6a) by $c^{\prime}$, averaging the results and summing the two equations. The result is the dynamic equation for the vertical flux of the passive tracer $J_{c}=\overline{w^{\prime} c^{\prime}}$ :

$\partial_{t} J_{c}=-\overline{w^{\prime 2}} \partial_{z} \bar{C}+g\left(\alpha \overline{\theta^{\prime} c^{\prime}}-\beta \overline{{S^{\prime} C^{\prime}}^{\prime}}\right)-\tau_{p c}^{-1} J_{c}$

where we have lumped together the pressure correlation and the dissipation terms in the dissipation-relaxation time scale $\tau_{\mathrm{pc}}$. Next, we must find an expression for the two covariances $\overline{\theta^{\prime} c^{\prime}}, \overline{S^{\prime} C^{\prime}}$ that enter Eq. (7). This is done by using the two equations for the fluctuating temperature and salinity fields which are obtained using a procedure analogous to the one used to arrive at (5). The results, which have been derived in the literature in many textbooks (e.g., Leslie, 1973; Lesieur, 1990), are:

$D_{t} \theta^{\prime}+u_{i}^{\prime} \partial_{i} \bar{T}+\partial_{i}\left(u_{i}^{\prime} \theta^{\prime}-\overline{u_{i}^{\prime} \theta^{\prime}}\right)=\chi_{\theta} \partial_{i}^{2} \theta^{\prime}$

$D_{t} s^{\prime}+u_{i}^{\prime} \partial_{i} \bar{S}+\partial_{i}\left(u_{i}^{\prime} s^{\prime}-\overline{u_{i}^{\prime} s^{\prime}}\right)=\chi_{s} \partial_{i}^{2} s^{\prime}$

where $\chi_{\theta, s}$ denote the heat and salt molecular diffusivities. We recall that $\chi_{\theta} \approx 100 \chi_{s}$ and that the ratio $v / \chi_{\theta} \approx 7$ is the Prandtl number for seawater. Multiply (8a) by $c^{\prime}$ and (5) by $\theta^{\prime}$, sum the two relations after averaging them and carry out an analogous operation with (8b). The result is the two equations:

$\partial_{t} \overline{\theta^{\prime} C^{\prime}}=-\left(J_{h} \bar{C}_{, z}+J_{c} \bar{T}_{, z}\right)-\tau_{c \theta}^{-1} \overline{\theta^{\prime} C^{\prime}}$

$\partial_{t} \overline{S^{\prime} C^{\prime}}=-\left(J_{s} \bar{C}_{, z}+J_{c} \bar{S}_{, z}\right)-\tau_{c s}^{-1} \overline{S^{\prime} C^{\prime}}$

where, as in Eq. (7), the two time scales $\tau_{\mathrm{c} \theta}$ and $\tau_{\mathrm{cs}}$ represent the relaxation-dissipation time scales of the corresponding correlations (for simplicity, we have neglected the third-order moments).

Before we proceed with the derivation, we pause momentarily to point out an important difference between passive and active tracers. Notice that Eqs. (9a) and (9b) are independent of the density and they are therefore quite general. Suppose however that $c^{\prime}$ had been an active scalar: if $c^{\prime}$ had been either $\theta^{\prime}, s^{\prime}$, Eqs. (9a) and (9b) would have given rise to the terms: 
$\overline{\theta^{\prime 2}}, \overline{S^{\prime 2}}$

as expected since they represent the potential energies stored in the $\theta$, s turbulent fields which are part of the energy budget; however, there is no such a term as $\overline{c^{\prime 2}}$ entering the above equations (even though it may be constructed from Eq. (5)) since a passive scalar field does not contribute to (affect) the energy balance. In the derivation of the mixing model presented in C10, the dynamic equations for (9c) were derived in Appendix A, Eqs. (A3) and (A4) of C10. In the $c^{\prime} \rightarrow \theta^{\prime}$ case, for example, Eq. (7) would become the equation for the heat flux and the second term would be twice the potential energy in the temperature field, representing a source of the heat flux. In such a case, Eq. (7) would coincide with Eq. (A1) of C10.

In Eqs. (9a) and (9b), $J_{h, s}$ are the vertical fluxes of heat and salt whose form was derived in $\mathrm{C} 10$ to be:

$J_{h}=\overline{W^{\prime} \theta^{\prime}}=-K_{h} \bar{T}_{, z}, \quad J_{s}=\overline{W^{\prime} S^{\prime}}=-K_{s} \bar{S}_{, z}$

where the heat and salt diffusivities $K_{h, s}$ are of the general form (2) and the corresponding $\Gamma_{h, s}$ are given in Appendix A. Taking the stationary limits in Eqs. (7), (9a), (9b) and using (9d), we finally obtain the desired form of the vertical flux of a passive tracer:

$J_{c}=\overline{W^{\prime} C^{\prime}}=-K_{c} \frac{\partial \bar{C}}{\partial z}, \quad K_{c}=\Gamma_{c} \frac{\varepsilon}{N^{2}}$,

where the mixing efficiency for the passive tracer is derived to be:

$\Gamma_{c}=\frac{p_{1}(\tau N)^{2}}{1+p_{1} p_{2}(\tau N)^{2}}\left(\frac{\overline{w^{\prime 2}}}{2 K}-p_{2} \Gamma_{\rho}\right)$

Relation (10b) deserves several comments. First, $\Gamma_{\rho}$ is the mixing efficiency of the buoyancy (density) field and is defined in terms of the heat-salt mixing efficiencies $\Gamma_{h, s}$ via the following relation:

$\Gamma_{\rho}=\left(\Gamma_{h}-R_{\rho} \Gamma_{s}\right)\left(1-R_{\rho}\right)^{-1}$,

where the $\Gamma_{h, s}$ are given by Eqs. (A1)-(A5) and (A9). Second, the determination of the variable $(\tau N)^{2}$ was discussed at length in Section 4.4 of $\mathrm{C} 10$ (for the $P=\varepsilon$ case) and we need only mention the final result presented in Eqs. (A10)-(A14). Third, the functions $p_{1,2}$ are defined as follows:

$p_{1} \equiv \frac{\tau_{p c}}{\tau}, \quad p_{2} \equiv \frac{\tau_{c \theta}}{\tau}=\frac{\tau_{c s}}{\tau}$,

whose determination is the most difficult part of the problem as discussed below. We recall that in $\mathrm{C10}$, we introduced the following dimensionless time scales:

$\pi_{1}=\frac{\tau_{p s}}{\tau}, \quad \pi_{4}=\frac{\tau_{p \theta}}{\tau}, \quad \pi_{2}=\frac{\tau_{s \theta}}{\tau}$,

which are given in terms of $R i$ and $R_{\rho}$ by relations (A13), (A14) which were assessed against NATRE data in Canuto et al. (2008, C8). Since relations (A13) show that the forms of $\pi_{1,4}$ conserve the heat-salt symmetry, there is no a priori justification to identify $p_{1}$ with either $\pi_{1,4}$ since in principle both are potentially acceptable candidates. The construction of the function:

$p_{1}=f\left(\pi_{1}, \pi_{4}\right)$

is a problem we tackle next. To do so, we use (A1) to transform (10b) to the following form:

$A_{c}=\frac{p_{1}}{1+p_{1} p_{2}(\tau N)^{2}}\left[1-p_{2}(\tau N)^{2} A_{\rho}\right]$.

Next, consider the active tracer salt in the limit in which the haline contraction coefficient becomes vanishingly small:

$\beta \sim(\partial \rho / \partial S)_{T} \rightarrow 0, \quad R_{\rho} \rightarrow 0$.
Physically, (13b) means that salinity is so diluted as not to affect the density, in which case salinity behaves like a passive scalar. In such a limit, we therefore expect that:

$\beta \rightarrow 0, \quad R_{\rho} \rightarrow 0: \quad K_{c} \rightarrow K_{s}$.

Furthermore, from (A5) and (A9) we have the following relations:

$\beta \rightarrow 0, \quad R_{\rho} \rightarrow 0: \quad r \rightarrow \infty, \quad p \rightarrow \pi_{4} \pi_{5}-\pi_{4} \pi_{2}, \quad q \rightarrow \pi_{1} \pi_{2}$.

At the same time, since the function $A_{\rho}$ has the same structure as (10c) w.r.t. heat and salt, we have the two additional relations:

$A_{\rho} \rightarrow A_{h} \rightarrow \frac{\pi_{4}}{1+\pi_{4} \pi_{5} x}, \quad x=(\tau N)^{2}\left(1-R_{\rho}\right)^{-1} \rightarrow(\tau N)^{2}$

where we used the first of (A4) and (13d). Introducing (13e) into (13a), a short algebra leads to the final form:

$R_{\rho} \rightarrow 0: \quad A_{c} \rightarrow \frac{p_{1}\left[1+\pi_{4}\left(\pi_{5}-p_{2}\right) x\right]}{\left(1+\pi_{4} \pi_{5} x\right)\left(1+p_{1} p_{2} x\right)}$

Next, we show how (13f) is related to the salt case. To do so, we employ both relations (A4) and (A5) and obtain:

$$
\begin{aligned}
A_{s} & =\frac{A_{h}}{r R_{\rho}}=\frac{A_{h}}{K_{h} / K_{s}}=A_{h} \frac{\pi_{1}(1+p x)}{\pi_{4}(1+q x)} \\
& \rightarrow \frac{\pi_{1}\left[1+\pi_{4}\left(\pi_{5}-\pi_{2}\right) x\right]}{\left(1+\pi_{4} \pi_{5} x\right)\left(1+\pi_{1} \pi_{2} x\right)},
\end{aligned}
$$

where in the last step we used (13d) for $p$ and $q$. Eq. (13g) coincides with (13f) provided we identify:

$\beta \rightarrow 0, \quad R_{\rho} \rightarrow 0: \quad p_{1}=\pi_{1}\left(R_{\rho}=0\right), \quad p_{2}=\pi_{2}\left(R_{\rho}=0\right)$.

Next, we treat the case when heat becomes passive which is represented by the following relations:

$\alpha \rightarrow 0, \quad R_{\rho} \rightarrow \infty: \quad K_{c} \rightarrow K_{h}$

In this limit, we have the following relations:

$$
\begin{aligned}
& p \rightarrow-\pi_{2} \pi_{4} R_{\rho}, \quad q \rightarrow \pi_{1}\left(\pi_{2}-\pi_{3}\right) R_{\rho}, \\
& x \rightarrow-\tau^{2} N^{2} R_{\rho}^{-1}, \quad p x \rightarrow \pi_{2} \pi_{4} \tau^{2} N^{2}, \quad q x \rightarrow-\pi_{1}\left(\pi_{2}-\pi_{3}\right) \tau^{2} N^{2}, \\
& r R_{\rho}=\frac{K_{h}}{K_{s}}=\frac{\pi_{4}}{\pi_{1}} \frac{1+q x}{1+p x} \rightarrow \frac{\pi_{4}}{\pi_{1}} \frac{1-\pi_{1}\left(\pi_{2}-\pi_{3}\right) \tau^{2} N^{2}}{1+\pi_{2} \pi_{4} \tau^{2} N^{2}} .
\end{aligned}
$$

The function $A_{h}$ defined in (A4) becomes:

$A_{h} \rightarrow \frac{\pi_{4}}{1+\pi_{2} \pi_{4}\left[1+\left(r R_{\rho}\right)^{-1}\right] \tau^{2} N^{2}}$

or, using the last of (14b) and after several algebraic steps, we obtain:

$A_{h} \rightarrow \frac{\pi_{4}\left[1-\pi_{1}\left(\pi_{2}-\pi_{3}\right) \tau^{2} N^{2}\right]}{1+\left(\pi_{1} \pi_{3}+\pi_{2} \pi_{4}\right) \tau^{2} N^{2}+\pi_{1} \pi_{2} \pi_{3} \pi_{4} \tau^{4} N^{4}}$

On the other hand, we also have that:

$A_{\rho} \rightarrow \frac{A_{h}}{r R_{\rho}}$.

Using (14d) and (14e), we now have that (13a) becomes:

$A_{c} \rightarrow \frac{p_{1}\left[1-\pi_{1}\left(p_{2}-\pi_{3}\right) \tau^{2} N^{2}\right]}{1+\left(\pi_{1} \pi_{3}+p_{1} p_{2}\right) \tau^{2} N^{2}+\pi_{1} \pi_{3} p_{1} p_{2} \tau^{4} N^{4}}$

Relations (14f) and (14d) coincide provided we identify:

$\alpha \rightarrow 0, \quad R_{\rho} \rightarrow \infty: \quad p_{1}=\pi_{4}\left(R_{\rho}=\infty\right), \quad p_{2}=\pi_{2}\left(R_{\rho}=\infty\right)$,

which is the second limit we shall use to construct relation (12b). 
Using the two relations (13h) and (14g), we suggest the following form for (12b):

$p_{1}=f\left(\pi_{1}, \pi_{4}\right)=\frac{R_{\rho}+R_{\rho}^{-1}}{R_{\rho} \pi_{4}^{-1}+R_{\rho}^{-1} \pi_{1}^{-1}}$

which we arrived at considering that since the shortest time scale dominates, one begins with the relation:

$\frac{1}{\tau_{p c}}=\frac{A}{\tau_{p s}}+\frac{B}{\tau_{p \theta}}$.

Using the $\pi$ 's from (12a) and $p_{1}$ from (11), we chose $A, B$ to satisfy the two requirements (13h) and (14g). The result is (15). As for $p_{2}$, its identification with $\pi_{2}$ seems unproblematic: $p_{2}=\pi_{2}$.

Using the variables $(\tau N)^{2}, \Gamma_{\rho}, p_{1,2}$ and (A2), (A3), in Figs. $1-3$ we plot $\Gamma_{c}$ given by (10b) for different values of $R i$ and $R_{\rho}$.

\section{Results}

In Fig. 1, corresponding to the doubly-stable, DS case, $R_{\rho}<0$, we present the dimensionless mixing efficiencies for a passive scalar, panel (a) and for spice, panel (b). The latter is defined as $\sigma \equiv \alpha$ $\theta^{\prime}+\beta s^{\prime}$ and since it is invariant under the exchange of the $T$ and $S$ fields, it might be considered as a possible candidate for a passive tracer. The corresponding mixing efficiency is given by

DS: $\mathrm{R}_{\rho}=0(\ldots),.-0.1(---),-1\left(\_\right),-10(-.-$.
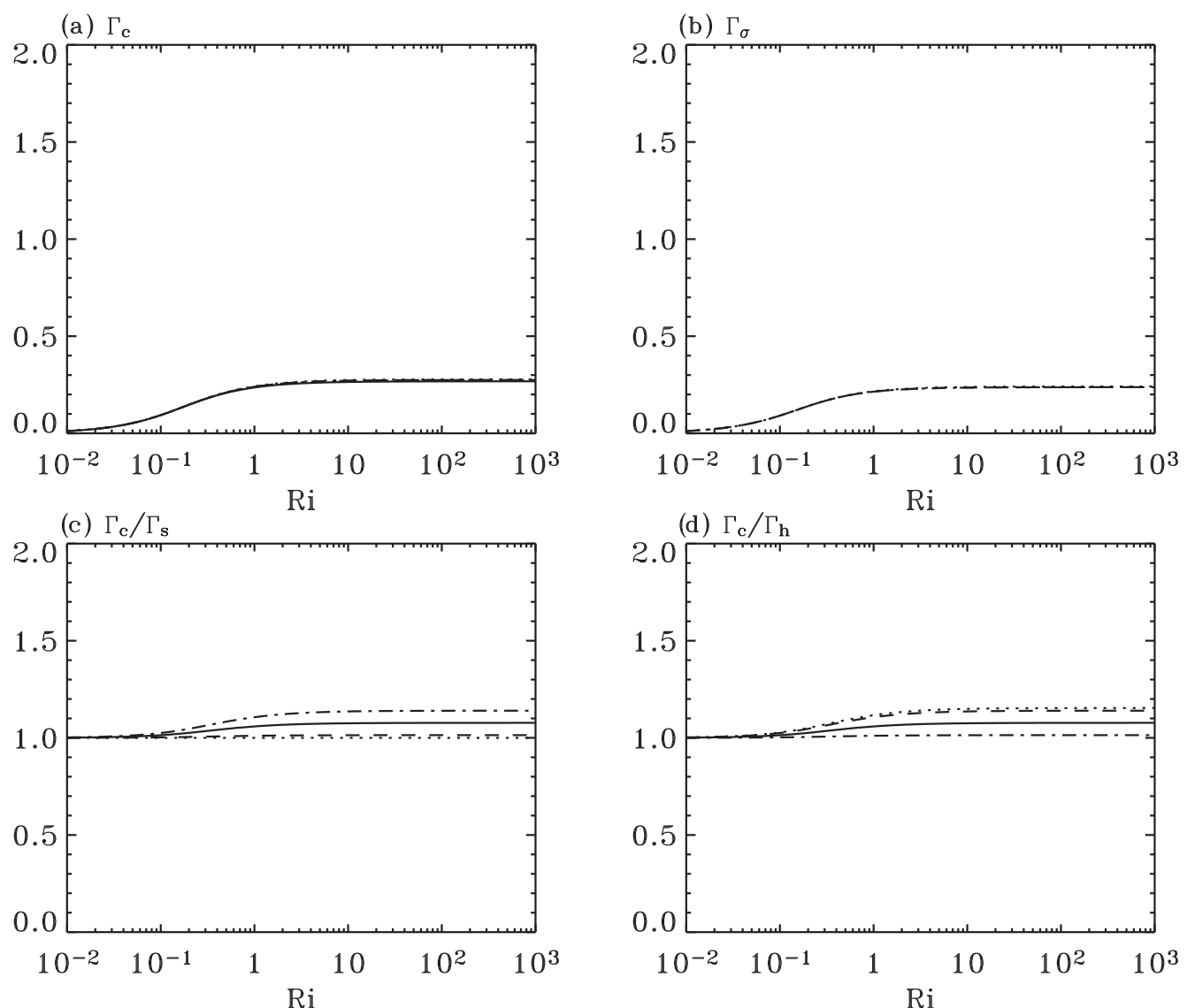

(d) $\Gamma_{\mathrm{c}} / \Gamma_{\mathrm{h}}$

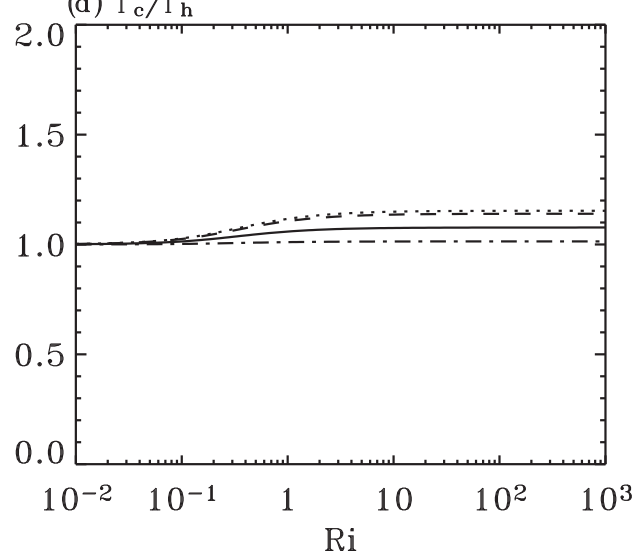

(e) $\Gamma_{\rho} / \Gamma_{\mathbf{c}}$

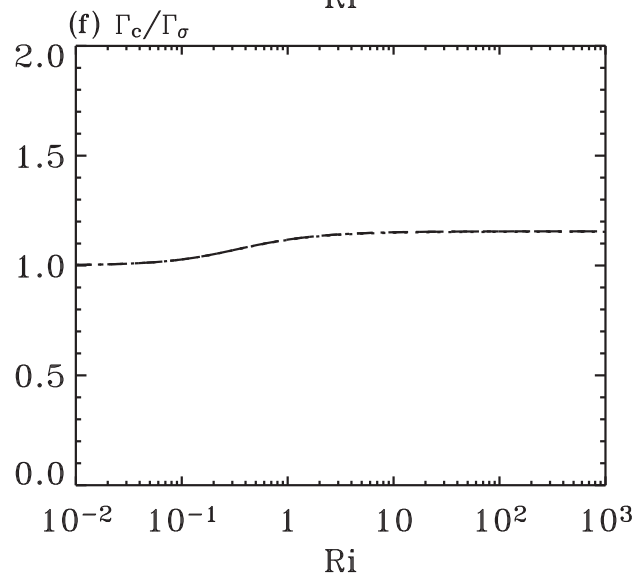

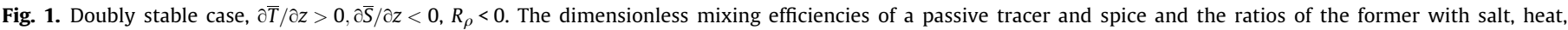
buoyancy and spice. Spice is defined as $\sigma \equiv \alpha \theta^{\prime}+\beta s^{\prime}$. 
DC: $\mathrm{R}_{\rho}=\infty(\ldots .),. 5(---), 1.67\left(\_\right), 1.25(-.-$.
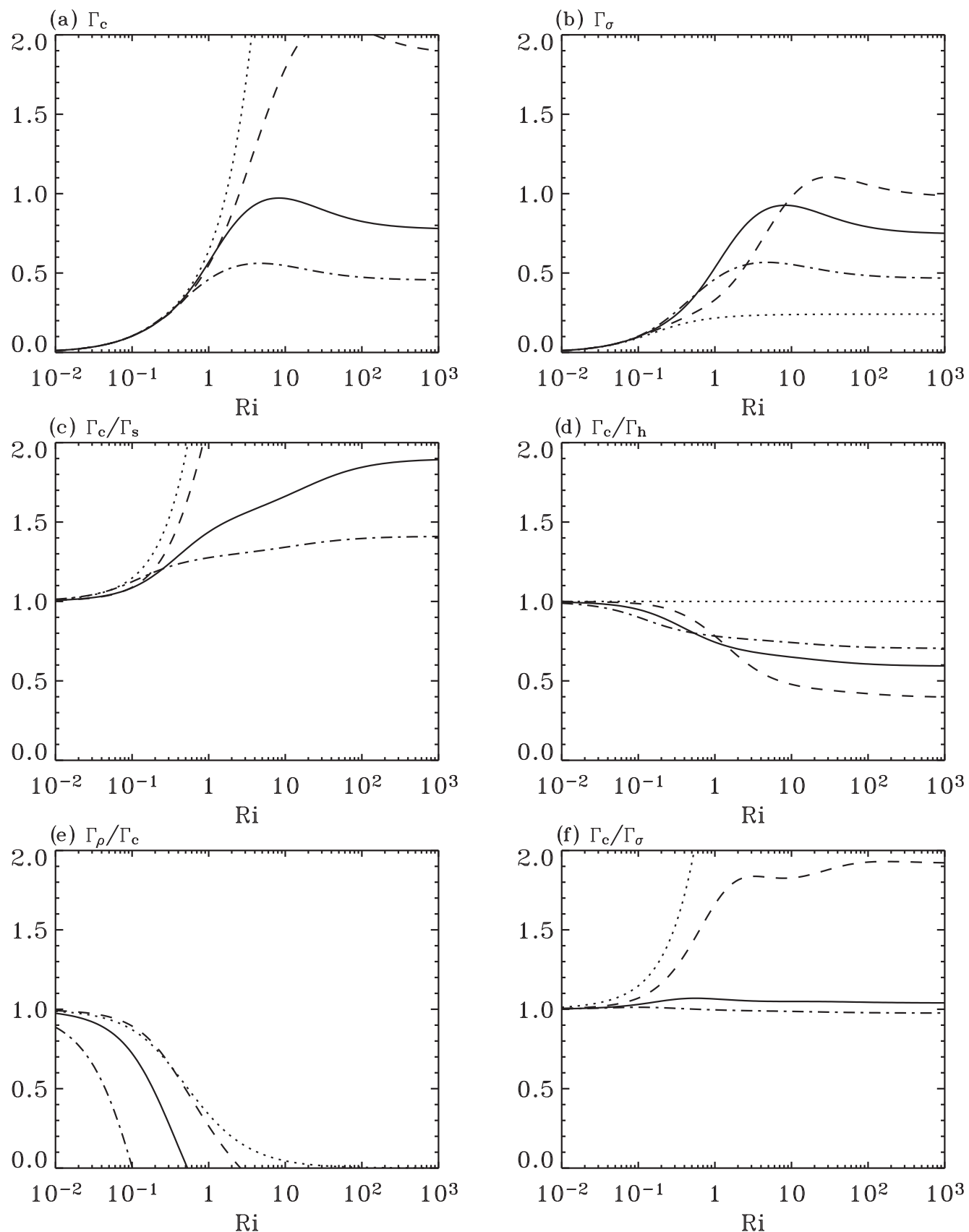

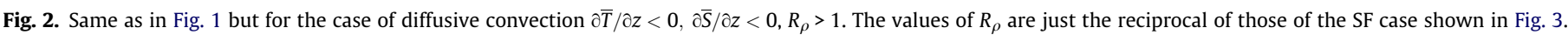

$\Gamma_{\sigma}=\left(\Gamma_{h}+R_{\rho} \Gamma_{s}\right)\left(1+R_{\rho}\right)^{-1}$

In the remaining panels (c)-(f) we exhibit the ratios of the passive tracer with salt, heat, buoyancy and spice. As one can observe from panels (c)-(f), to all practical purposes, there is little difference between the diffusivity of a passive scalar and that of salt, heat or spice. In principle, this might be construed as a justification of the common assumption that a passive tracer diffusivity can be identified with that of salt. However, the Southern Ocean which is the largest $\mathrm{CO}_{2}$ sink due to its largest extent (Takahashi et al., 2009), is not primarily characterized by $R_{\rho}<0$ but by diffusive convection, $\mathrm{DC}, R_{\rho}>1$. Therefore, in carbon-cycle studies, identifying the passive tracer with salt may lead to incorrect results. The DC results are presented in Fig. 2. As one can observe from panel (c), the passive tracer diffusivity is larger than that of salt up to a factor of two and it is smaller than that of heat by the same amount, panel (d). Finally, in Fig. 3 we present the results for the SF case, in which case we notice that the tracer mixing efficiency is smaller than that of salt but larger than that of heat by roughly the same amount.

\section{Assessment of the model prediction against the NATRE data}

Ledwell et al. (1993, 1998) reported passive tracer diffusivity measurements during the NATRE $(26 \mathrm{~N}, 28 \mathrm{~W})$ experiment. In Fig. 4 we reproduce their data (open triangles) at a depth of $300 \mathrm{~m}$ for two successive periods (the point on the left corresponds 
$\mathrm{SF}: \mathrm{R}_{\rho}=0(\ldots),. 0.2(---), 0.6\left(\_\right), 0.8(-.-$.
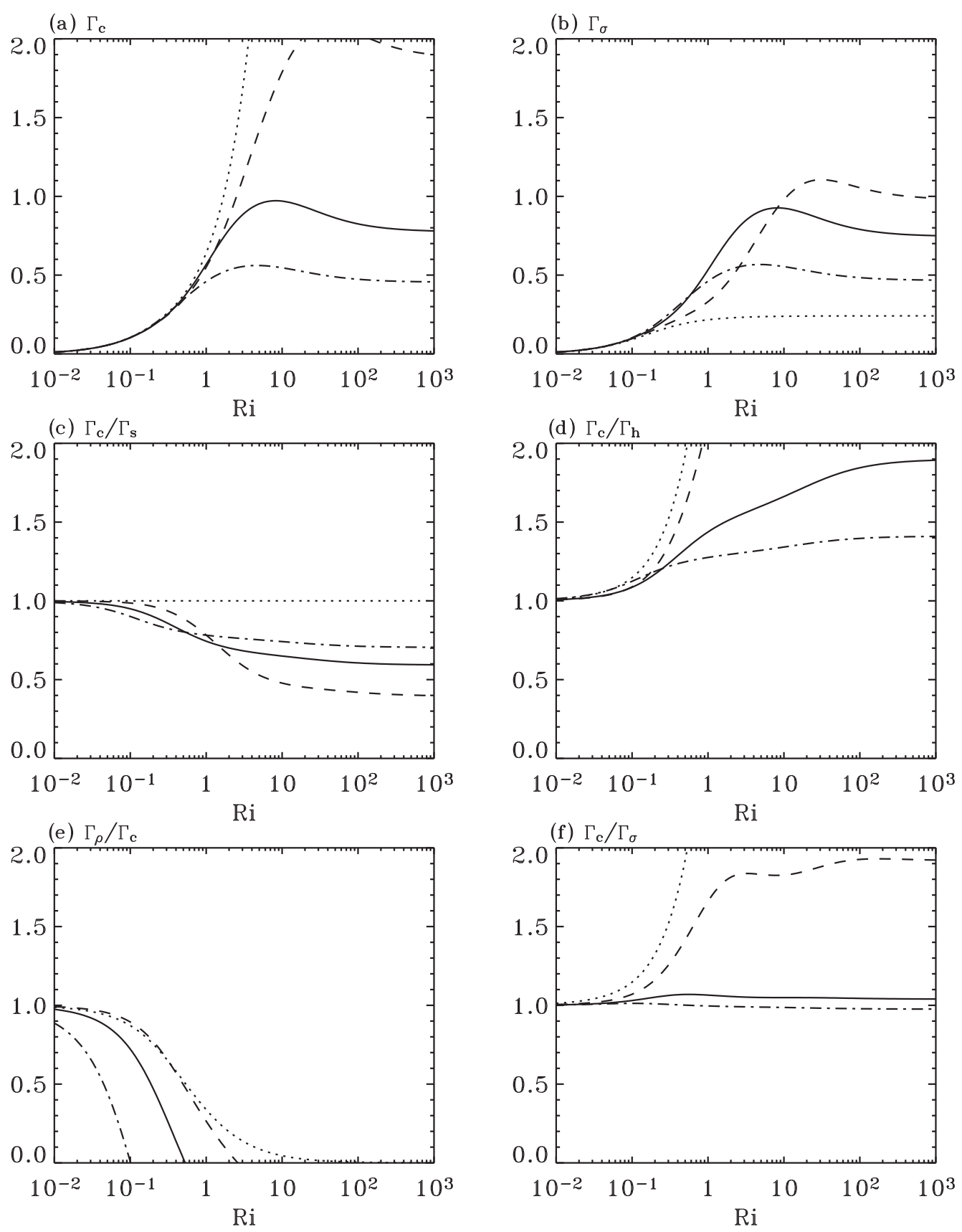

Fig. 3. Same as in Fig. 1 salt fingers, $\partial \bar{T} / \partial z>0, \partial \bar{S} / \partial z>0, R_{\rho}<1$.

to the first 6 months' measurements while the one on the right corresponds to the subsequent 24 months). The model results (denoted by a cross) are closer to the data of the first measurement. This may be due to the fact that the values of $R_{\rho}$ used to compute the model results (see description below) were obtained from St. Laurent and Schmitt (1999), cited as SS99 and corresponded to a time closer to the measurement of the first period. Since we have no data other than those at $300 \mathrm{~m}$, the $z$-profiles must be viewed as theoretical predictions to be assessed when future measurements will become available. The reason why the model results (crosses) exhibit an error bar is because they are based on the measured values of $R_{\rho}$ which have errors.

As suggested by a referee, in the same figure we also plot the $z$ profile of the salt diffusivity $K_{s}$ (black squares). As in the tracer case, the model salt diffusivities have an error bar because they depend on the measurements based values of $R_{\rho}$. As expected on the grounds of Fig. 3(c), in a salt-fingers regime such a one at NATRE, one expects to have $K_{s}>K_{c}$ which is indeed the case in Fig. 4.

It is important to discuss how the model results (crosses and black squares) were obtained without the use of an OGCM. We employed the following procedure. The $z$-profile of the density ratio $R_{\rho}$ was taken from the NATRE data from SS99. The functions $A_{h, s}$ were constructed using (A4) in which the heat to salt flux ratio " $r$ " was computed from its definition (A5). The variable $x$, defined in (A9), was obtained by solving Eqs. (A10), (A11), (A12); the variable $\overline{w^{\prime 2}} / K$ is given by Eqs. (A.2) and (A3). As for the Richardson number, while in $\mathrm{C} 10$ its background value $R i(\mathrm{bg})$ in the thermocline was taken to be 0.5 , we have since then found that an $R_{\rho}$ 


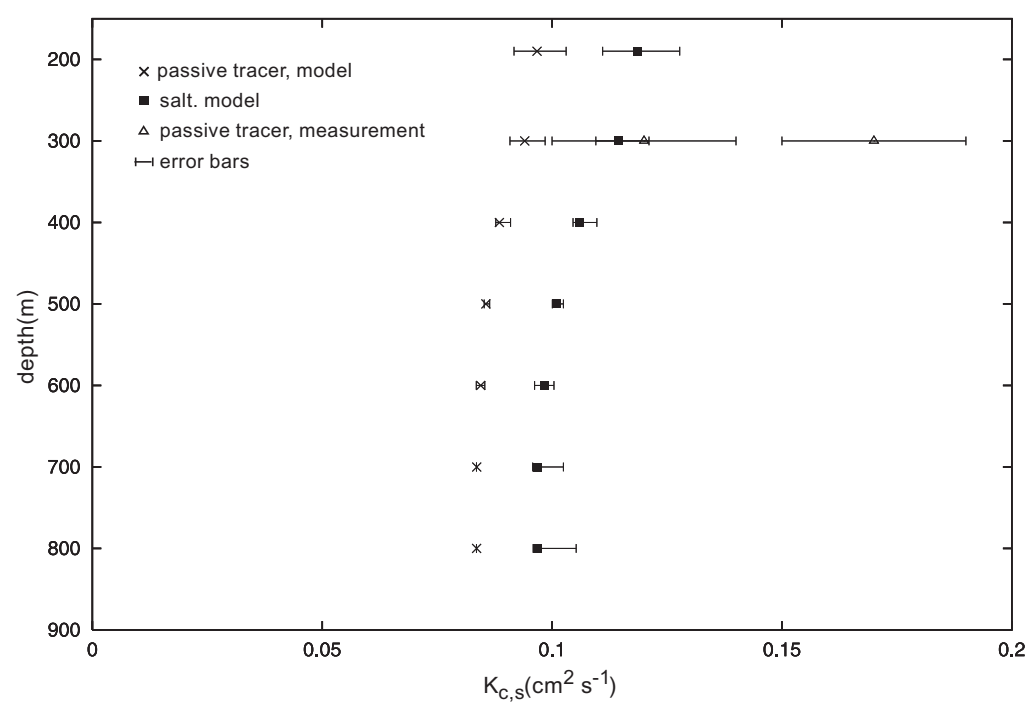

Fig. 4. The $z$-profile of the model passive tracer and salinity diffusivities and the NATRE measurements. For details, see Section 4.

dependent $R i(b g)$ yields a better fit to the SS99 data on heat and salt diffusivities. Since we believe that $R i(b g)$ is set by a self-limiting process in which the turbulent viscosity produced by the wave breaking interferes with the waves themselves, we tried the hypothesis that in the background $\Gamma_{m}$, which is proportional to the viscosity, is a constant. Since $\Gamma_{m}$ is a function of $R i$ and $R_{\rho}$, adopting this hypothesis yields $R i(b g)$ vs. $R_{\rho}$. The best fit to the SS99 data was obtained with $\Gamma_{m}=0.46$ and the Ri(bg) corresponding to that value was accordingly used.

\section{Maps of active and passive tracer diffusivities}

In Figs. 5-10 we present meridional maps, depth vs. latitude (at a longitude of $29 \mathrm{~W}$ passing through the NATRE location), of the vertical diffusivities $\left(\mathrm{cm}^{2} \mathrm{~s}^{-1}\right)$ for the following fields:

momentum, heat, salt, spice, buoyancy, passive scalar.

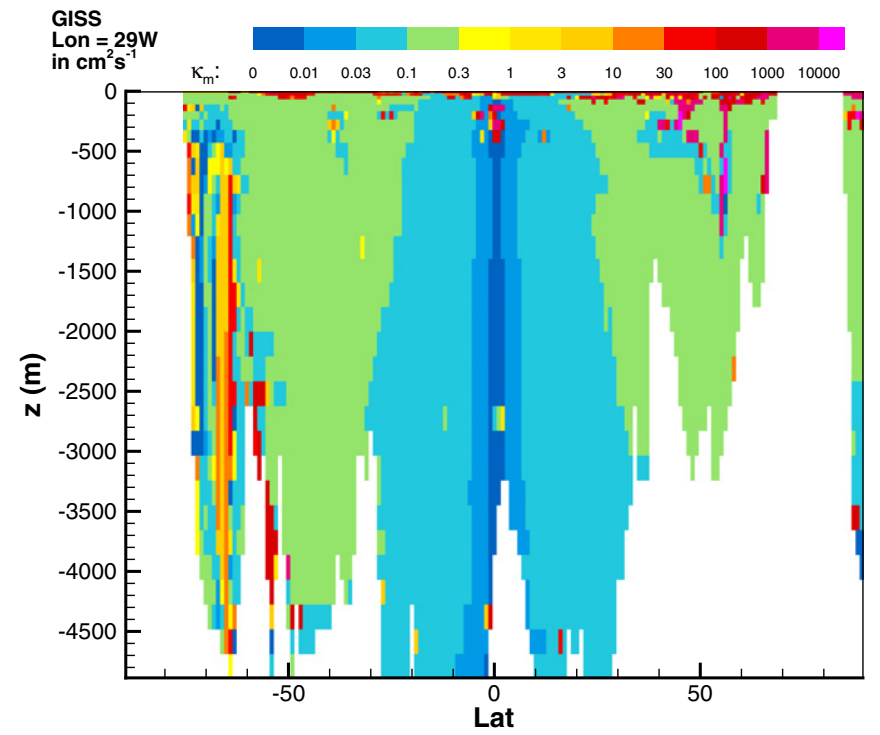

Fig. 5. Depth-latitude maps of the momentum diffusivity at $29 \mathrm{~W}$ (near the NATRE location). For comments see Section 5.

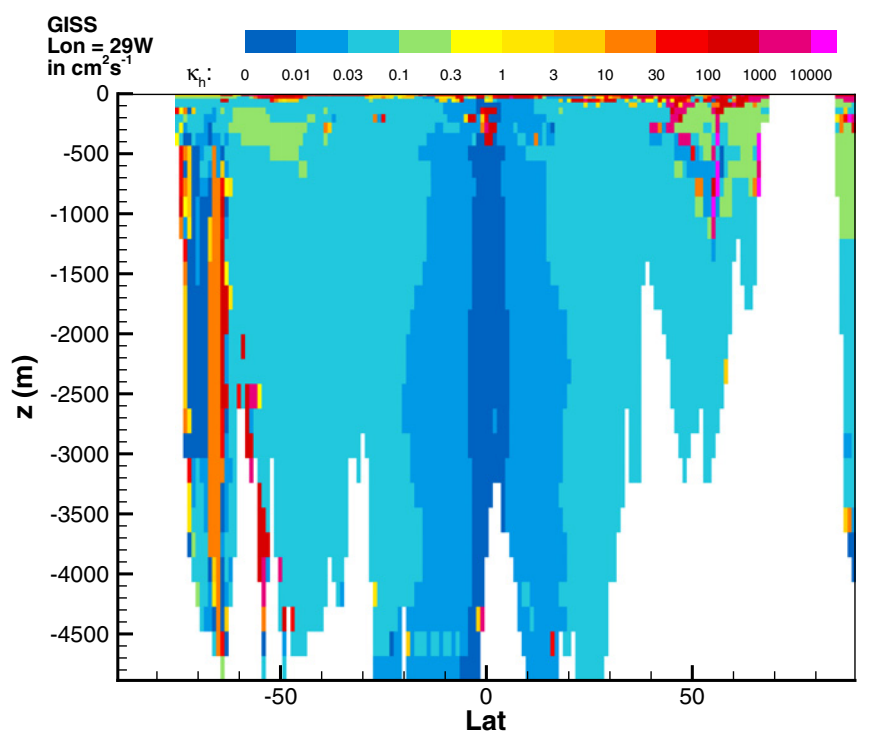

Fig. 6. Same as Fig. 5 but for the heat diffusivity.

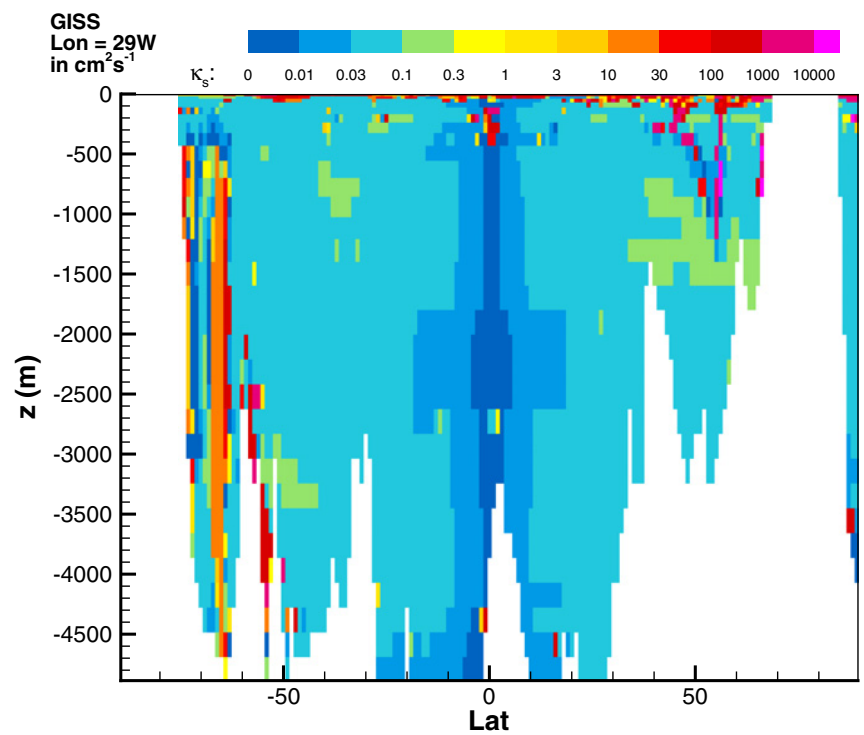

Fig. 7. Same as Fig. 5 but for the salt diffusivity. 


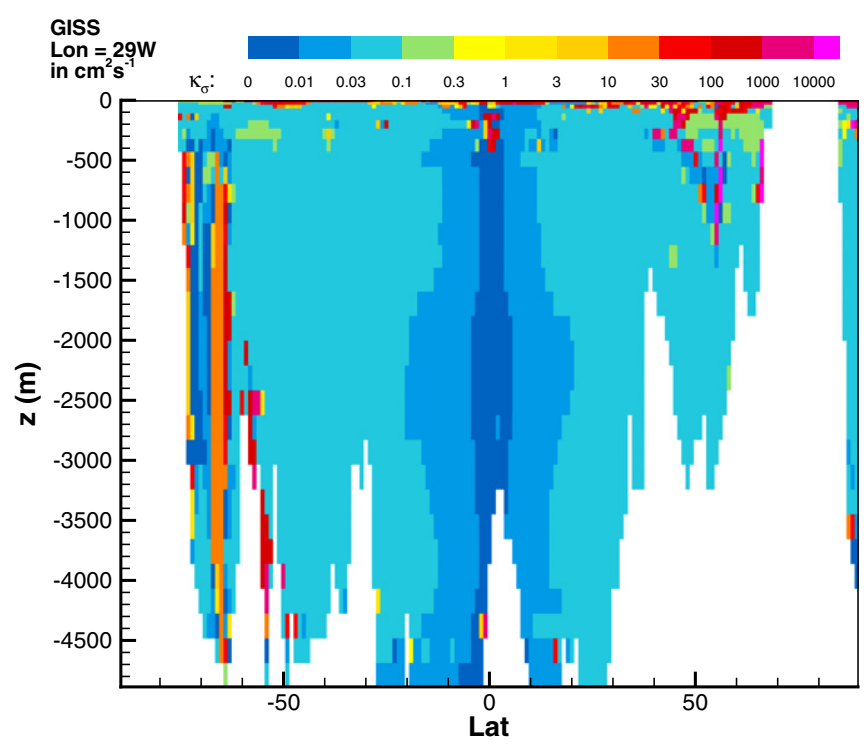

Fig. 8. Same as Fig. 5 but for the spice $\sigma \equiv \alpha \theta^{\prime}+\beta s^{\prime}$ diffusivity.

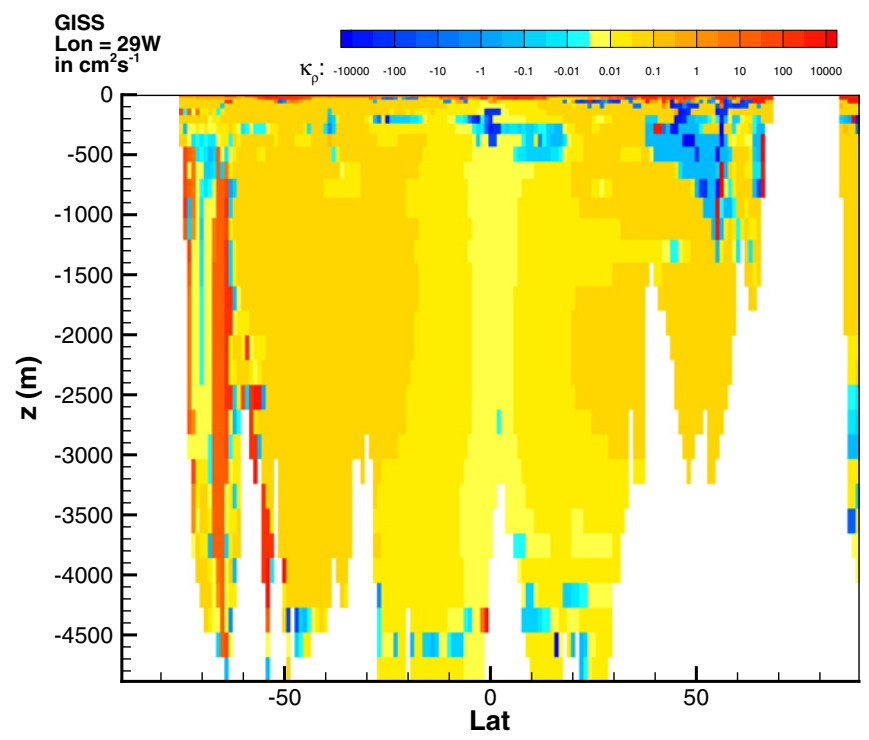

Fig. 9. Same as Fig. 5 but for the buoyancy diffusivity.

The vertical mixing model is given in $\mathrm{C} 10$ except that the Ri(bg) was determined as discussed in Section 4. The inputs to the mixing model (velocity, temperature and salinity fields) were obtained from a snapshot of existing model output from a coupled oceanatmosphere general circulation model, specifically, the GISS coupled Model E with $1^{\circ} \times 1.25^{\circ}$ horizontal resolution and 32 vertical levels. The snapshot was taken after 150 years of model spin-up in which the KPP vertical mixing scheme had been used. Several comments are in order: in all cases, the large values found near the top correspond to the enhanced diffusivities in the mixed layer. For all the diffusivities, the low values at the equator are due to the latitude dependence of the breaking of the IGW (internal gravity waves). As expected in a stably stratified case, the momentum diffusivity, Fig. 5, is larger than both heat Fig. 6 and salt Fig. 7 by a sizeable but not uniform, factor: the momentum diffusivities are between $0.1-0.3$ while the heat diffusivity is at most 0.1 . Salt diffusivities are larger than heat in places where SF (salt fingers) operate, as seen by the green patches in Fig. 7. Much of the effect of DD is however

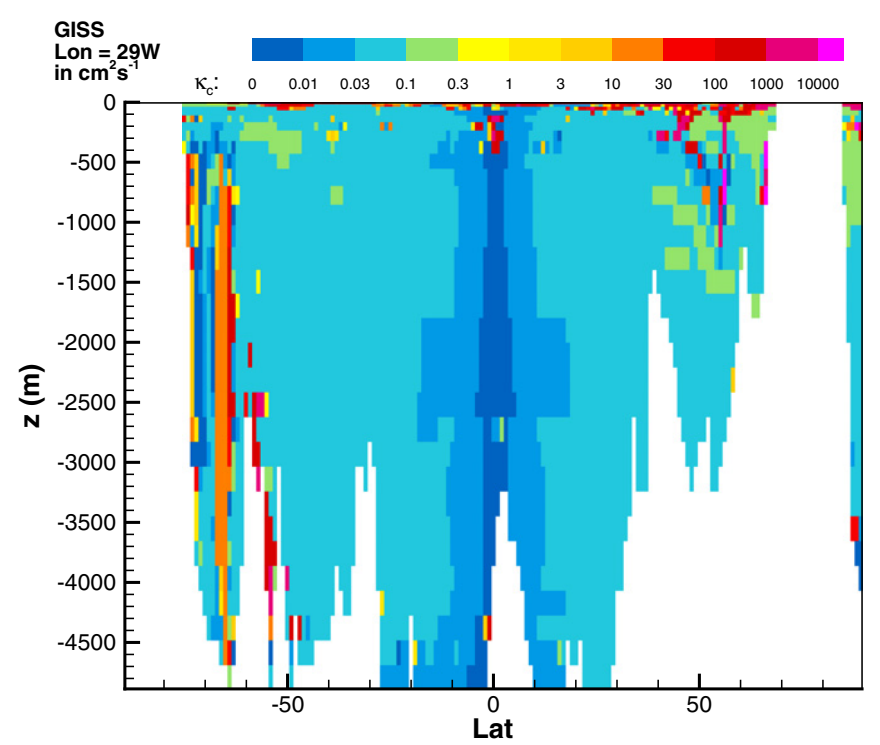

Fig. 10. Same as Fig. 5 but for the passive scalar diffusivity.

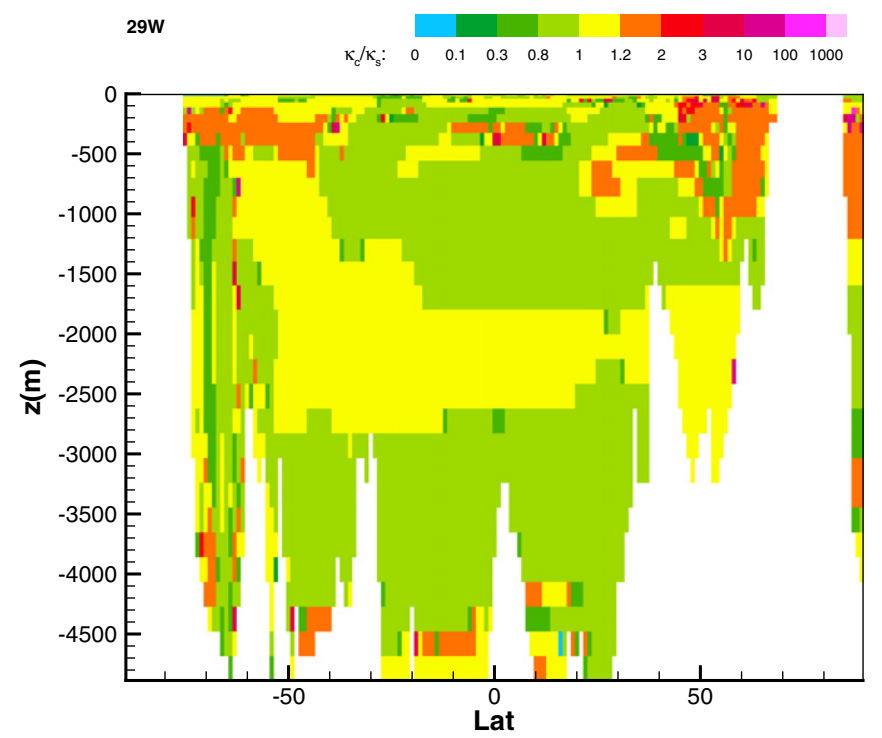

Fig. 11. Ratio of the passive scalar to salt diffusivity in the same region as in Figs. 510. For comments see Section 5.

washed out in the case of spice, Fig. 8, whose diffusivity is more similar to that of heat than salt. In Fig. 9 we exhibit the diffusivity $K_{\rho}$, Eqs. (2) and (10c), corresponding to buoyancy. Since in regions of strong SF or DC such a diffusivity is negative, one can observe the patches where that occurs. For the vast majority of ocean regimes, the values are, as expected, smaller than those of heat and salt separately. In Fig. 10 we present the passive scalar diffusivity that can be compared with the heat and salt in Figs. 6 and 7. To make the comparison easier, in Figs. 11 and 12 we exhibit the ratios of the passive tracer diffusivity to those of salt and heat. The green regions in Fig. $11, \kappa_{c}<\kappa_{s}$, and the red regions, $\kappa_{c}>\kappa_{h}$, in Fig. 12, correspond to a SF regime (see Eq. (19)). The yellow regions correspond to doubly stable regimes in which the passive scalar is very close to heat and salt, as seen in Fig. 1(c)-(d). To further visualize the results of the model, in Figs. 13 and 14 we exhibit the momentum and heat diffusivities of the KPP model (Large et al., 1994) which can be compared with Figs. 5 and 6, respectively. The different texture of the results is apparent. 


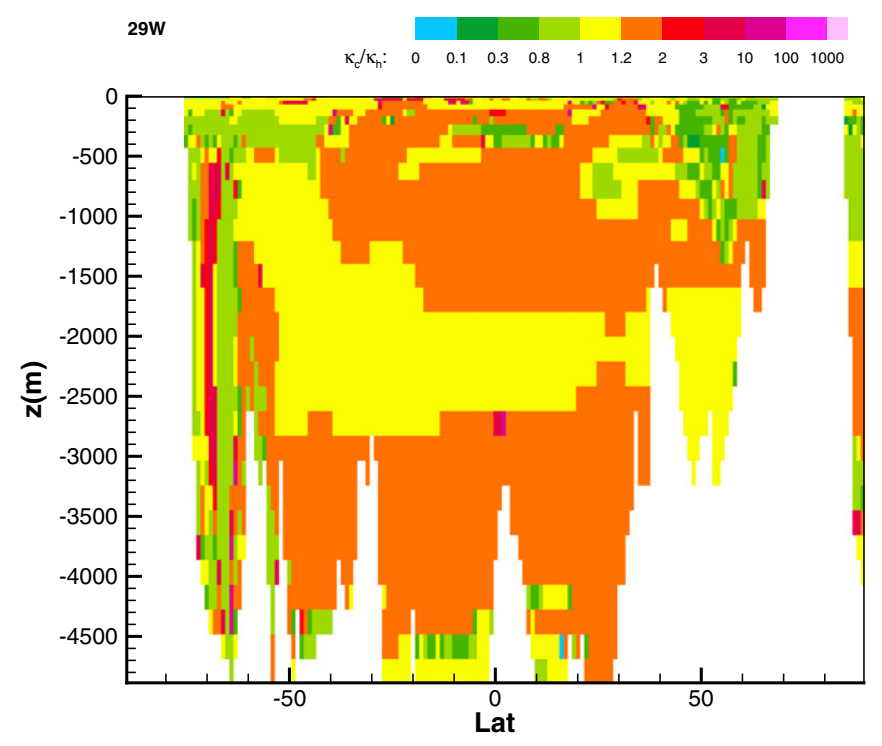

Fig. 12. Same as Fig. 11 but for the ratio of passive scalar to heat diffusivity.

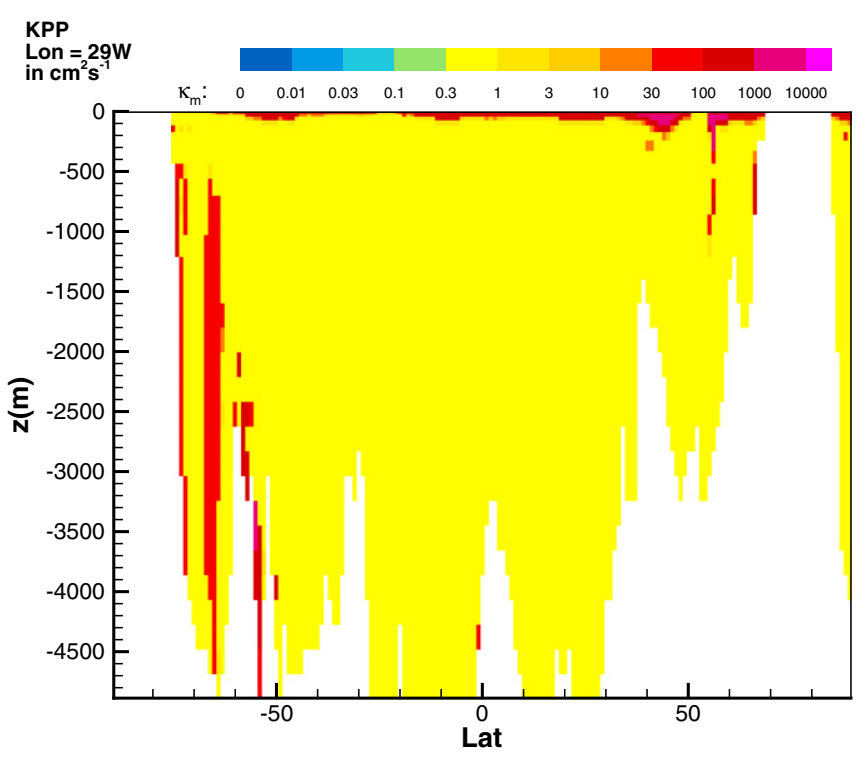

Fig. 13. Momentum diffusivity in the KPP model. Notice the different texture of the results from Fig. 5.

\section{Conclusion}

In this paper we have derived a general expression for the diffusivity of a passive tracer in terms of large scale variables $R i$ and $R_{\rho}$. The result is given by relations (10a), (10b). The most relevant conclusion of this work is that the common identification of a tracer diffusivity with that of salt is valid only in regions of doubly stable (DS) T-S gradients which does not occur in much of the Southern Ocean where the largest $\mathrm{CO}_{2}$ absorption occurs. In those regions, the dominant feature is an unstable T-gradient and a stable S-gradient, a regime known as Diffusive Convection, DC, as the maps (courtesy of D.E. Kelly) shown in Figs. 6 and 7 of C10 show. The results in Fig. 2 show that in the DC regime the tracer diffusivity for values of Ri of order unity, is roughly twice as large as that of salt and twice as small as that of heat. Conversely, in the SF case, the tracer diffusivity for values of $R i$ of order unity, is roughly twice

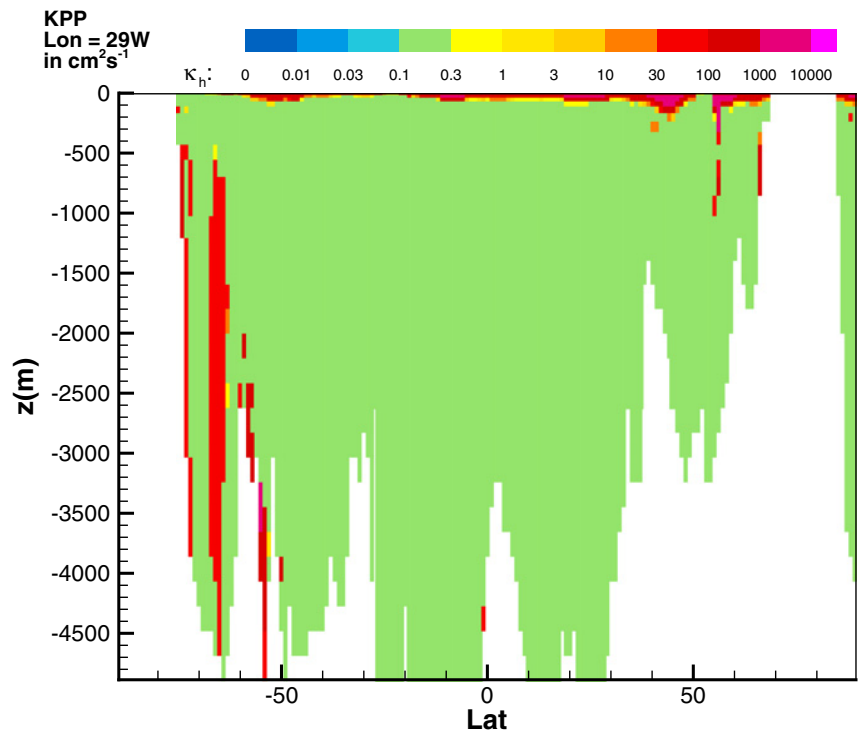

Fig. 14. Heat diffusivity in the KPP model. The different texture of the results from those in Fig. 6 is apparent.

as small as that of salt and twice as large as that of heat, a situation that can be summarized as follows:

DS : $K_{c} \approx K_{h} \approx K_{s}$,

DC : $K_{c}>K_{s}, \quad K_{c}<K_{h}$,

$\mathrm{SF}: K_{c}<K_{s}, \quad K_{c}>K_{h}$.

The next step is to employ relation (10b), together with the vertical mixing scheme in $\mathrm{C} 10$, in an OGCM with a carbon-cycle model and assess the difference with the previous assumption of a tracer represented by salt.

\section{Acknowledgements}

The authors would like to thank two anonymous referees who asked us very interesting questions the answers to which helped improve the original manuscript.

\section{Appendix A. Ingredients of the model}

General relations:

$\Gamma_{\alpha}=\frac{1}{2}(\tau N)^{2} A_{\alpha} \frac{\overline{W^{\prime 2}}}{K}, \quad S_{\alpha}=A_{\alpha} \frac{\overline{w^{\prime}}}{K}$.

Ratio $\overline{w^{\prime 2}} / 2 K$. From Eqs. (37) and (39) of C10 it follows that: General case:

$\overline{\frac{W^{\prime 2}}{2 K}}=\frac{1}{3}\left[1+\frac{2}{15} A_{\rho}(\tau N)^{2}+\frac{1}{10} A_{m}(\tau \Sigma)^{2}\right]^{-1}$,

which, for the production $=$ dissipation case, simplifies to:

$P=\varepsilon: \frac{\overline{w^{\prime 2}}}{2 K}=\left[\frac{30}{7}+A_{\rho}(\tau N)^{2}\right]^{-1}$.

Heat and salt:

$A_{h}=\pi_{4}\left[1+p x+\pi_{4} \pi_{2} x\left(1-r^{-1}\right)\right]^{-1}, \quad A_{s}=A_{h}\left(r R_{\rho}\right)^{-1}$.

Heat-to-salt flux ratio:

$r \equiv \frac{\alpha_{T} \overline{w \theta}}{\alpha_{s} \overline{w s}}=\frac{1}{R_{\rho}} \frac{K_{h}}{K_{s}}=\frac{\pi_{4}}{\pi_{1}} \frac{1}{R_{\rho}} \frac{1+q x}{1+p x}$, 
Momentum:

$A_{m}=\frac{A_{m 1}}{A_{m 2}}$,

$A_{m 1}=\frac{4}{5}-\left[\pi_{4}-\pi_{1}+\left(\pi_{1}-\frac{1}{150}\right)\left(1-r^{-1}\right)\right] x A_{h}$,

$A_{m 2}=10+\left(\pi_{4}-\pi_{1} R_{\rho}\right) x+\frac{1}{50}(\tau \Sigma)^{2}$,

Dimensionless variables $x, p$ and $q\left(N^{2}=-g \rho_{0}^{-1} \partial \bar{\rho} / \partial z\right)$ :

$x=(\tau N)^{2}\left(1-R_{\rho}\right)^{-1}, \quad p=\pi_{4} \pi_{5}-\pi_{4} \pi_{2}\left(1+R_{\rho}\right), \quad q$

$=\pi_{1} \pi_{2}\left(1+R_{\rho}\right)-\pi_{1} \pi_{3} R_{\rho}$

Cubic equation for $G_{m} \equiv(\tau \Sigma)^{2}=(\tau N)^{2} R^{-1}$ :

$c_{3} G_{m}^{3}+c_{2} G_{m}^{2}+c_{1} G_{m}+1=0$,

with:

$c_{3}=A_{1} R i^{3}+A_{2} R i^{2}, \quad c_{2}=A_{3} R i^{2}+A_{4} R i, \quad c_{1}=A_{5} R i+A_{6}$,

where:

$$
\begin{aligned}
& 150\left(1-R_{\rho}\right)^{3} A_{1}=\pi_{1} \pi_{4}\left(\pi_{4}-\pi_{1} R_{\rho}\right)\left\{\pi_{2}\left(15 \pi_{3}+7\right)\left(R_{\rho}^{2}+1\right)\right. \\
& \left.\quad+\left[14\left(\pi_{2}-\pi_{3}\right)-15 \pi_{3}^{2}\right] R_{\rho}\right\} \\
& 9000\left(1-R_{\rho}\right)^{2} A_{2}=\pi_{1} \pi_{4}\left\{\pi_{2}\left(210 \pi_{1}-150 \pi_{3}+7\right)\left(R_{\rho}^{2}+1\right)\right. \\
& \left.\quad+\left[14\left(\pi_{2}-\pi_{3}\right)\left(1+15 \pi_{1}+15 \pi_{4}\right)+150 \pi_{3}^{2}\right] R_{\rho}+210 \pi_{2}\left(\pi_{4}-\pi_{1}\right)\right\} \\
& 150\left(1-R_{\rho}\right)^{2} A_{3}=\pi_{1}\left[5 \pi_{2} \pi_{4}\left(30 \pi_{3}+17\right)+\pi_{1}\left(15 \pi_{3}+7\right)\right] \\
& \quad\left(R_{\rho}^{2}+1\right)-\left(15 \pi_{3}+7\right)\left(\pi_{1}^{2}-\pi_{4}^{2}\right)-\left[10 \pi_{1} \pi_{3} \pi_{4}\left(15 \pi_{3}+17\right)\right. \\
& \left.\quad+15 \pi_{2}\left(\pi_{1}^{2}+\pi_{4}^{2}\right)+14 \pi_{1} \pi_{4}\left(1-10 \pi_{2}\right)\right] R_{\rho} \\
& 9000\left(1-R_{\rho}\right) A_{4}=\left[150\left(\pi_{1} \pi_{3}+\pi_{2} \pi_{4}\right)-7 \pi_{1}\left(1+30 \pi_{1}\right)\right] \\
& \quad R_{\rho}-150\left(\pi_{1} \pi_{2}+\pi_{3} \pi_{4}\right)+7 \pi_{4}\left(1+30 \pi_{4}\right) \\
& 30\left(1-R_{\rho}\right) A_{5}=\left[-30\left(\pi_{1} \pi_{3}+\pi_{2} \pi_{4}\right)-17 \pi_{1}\right] R_{\rho} \\
& \quad+30\left(\pi_{1} \pi_{2}+\pi_{3} \pi_{4}\right)+17 \pi_{4}, \quad A_{6}=-1 / 60
\end{aligned}
$$

The $\pi$ 's: For $R_{\rho} \geqslant 0$ and $R i \geqslant 0$ :

$$
\begin{aligned}
& \pi_{1}=\pi_{1}^{0}\left(1+\frac{R i}{1+a R_{\rho}^{-1}}\right)^{-1}, \quad \pi_{4}=\pi_{4}^{0}\left(1+\frac{R i}{1+a R_{\rho}}\right)^{-1}, \\
& \pi_{2}=\pi_{2}^{0}(1+R i)^{-1}\left[1+2 R i R_{\rho}\left(1+R_{\rho}^{2}\right)^{-1}\right], \quad \pi_{5}=\pi_{5}^{0}, \\
& \pi_{1}^{0}=\pi_{4}^{0}=\left(27 K o^{3} / 5\right)^{-1 / 2}\left(1+\sigma_{t}^{-1}\right)^{-1}, \\
& \pi_{2}^{0}=1 / 3, \quad \pi_{3}=\pi_{3}^{0}=\pi_{5}^{0}=\sigma_{t}=0.72 .
\end{aligned}
$$

where $a=10$. For $R_{\rho}<0$ and $R i>0$, we have:

$\pi_{1,4}=\pi_{1,4}^{0}(1+R i)^{-1}, \quad \pi_{2,3,5}=\pi_{2,3,5}^{0}$.

For $R i<0, \pi_{k}=\pi_{k}^{0}$ for any $k$. In relations (A13) $K o=1.6$ is the Kolmogorov constant.

\section{References}

Canuto, V.M., Cheng, Y., Howard, A.M., 2008. A new model for double diffusion + turbulence. Geophys. Res. Lett. 35, L02613. doi:10.1029/ 2007GL032580. Cited as C8.

Canuto, V.M., Howard, A.M., Cheng, Y., Muller, C.J., Leboissetier, A., Jayne, S.R., 2010. Ocean turbulence III: new GISS vertical mixing scheme. Ocean Modell. 34, 7091. Cited as C10.

Gregg, M.C., Winkel, D.P., Sanford, T.S., Peters, H., 1996. Turbulence produced by internal waves in the ocean thermocline at mid and low latitudes. Dynam. Atmos. Oceans 24, 1-14.

Kunze, E., Sanford, T.S., 1996. Abyssal mixing: where it is not. J. Phys. Oceanogr. 26, 2286-2296.

Large, W.G., McWilliams, J.C., Doney, S.C., 1994. Ocean vertical mixing: a review with non-local boundary layer parameterization. Rev. Geophys. 32, 363-403.

Ledwell, J.R., Watson, A.J., Law, C.S., 1993. Evidence for slow mixing across the pycnocline from open ocean tracer release experiment. Nature 364, 701-703.

Ledwell, J.R., Watson, A.J., Law, C.S., 1998. Mixing of a tracer in the pycnocline. J. Geophys. Res. 103, 21499-21529.

Lesieur, M., 1990. Turbulence in Fluids. Kluwer Academic Publishers, Dordrecht. 412pp.

Leslie, D.C., 1973. Developments in the Theory of Turbulence. Clarendon Press, Oxford. 368pp.

Polzin, K., Toole, J.M., Schmitt, R.W., 1995. Finescale parameterization of turbulent dissipation. J. Phys. Oceanogr. 25, 306-328.

Polzin, K., 1996. Statistics of the Richardson number, mixing models and fine structure. J. Phys. Oceanogr. 26, 1409-1425.

Schmid, M., Busbridge, M., Wüest, A., 2010. Double-diffusion convection in Lake Kivu. Limnol. Oceanogr. 55 (1), 225-238.

St. Laurent, L., Schmitt, R.W., 1999. The contribution of salt fingers to vertical mixing in the north atlantic tracer release experiment. J. Phys. Oceanogr. 29, 14041424.

Takahashi, T. et al., 2009. Climatological mean and decadal change in surface ocean $\mathrm{pCO}_{2}$ and net sea-air flux over the global oceans. Deep Sea Res. II 56, 554-577.

Toole, J.M., 1998. Turbulent mixing in the ocean. In: Chassignet, E.P., Verron, J. (Eds.), Ocean modeling and parameterization, NATO, ASI, Series C, vol. 516. Kluwer Academic Publishers, MA, USA, pp. 171-190.

UNESCO, 1981. 10th Report of the joint panel on oceanographic tables and standards. UNESCO Tech. Papers in Marine Science No. 36, Paris. 